\title{
Research on the Shearing Performances of PBL Connector in Non-Steel-Cell Joint Section of Steel-Concrete Hybrid Girder Bridge
}

\author{
Jia HE ${ }^{1, a}$, Qin CAI ${ }^{2, b}$, Lin-lin LI ${ }^{3, c}$ \\ ${ }^{1}$ College of civil engineering \& architecture, China Three Gorges University, No. 8 University Road \\ in Yichang, China \\ 2 Yichang investment and development co., Hubei traffic investment group co., LTD, Development \\ avenue Water-Yue city office 21 layer in Yichang, China \\ ${ }^{3}$ Hubei Transportation Planning and Design Institute, No. 7 Long Yang Road in Han yang District in \\ Wuhan, China \\ a244037253@qq.com, b25142478@qq.com, c30793819@qq.com
}

Key words: hybrid girder bridge; non-steel-cell joint section; PBL connector; shear resistance Abstract: Non-steel-cell joint section is a common way to connect the steel beam and the concrete beam in steel-concrete hybrid girder bridge, and the PBL connector with large rigidity is usually adopted in non-steel-cell joint section. Shear resistance of PBL connector determines the success of joint force transmission. But the PBL connection in different structures has different boundary conditions and the state of stress. By means of the finite element numerical analysis method, the different failure modes of the steel roof and the bottom plate PBL connector under ultimate bearing capacity load were analyzed. The results show that the concrete stress level of compressive zone of columns hole is relatively balanced; the stress value of concrete in tensile stress area of connector close to bearing plate hole is bigger, and the stress value of concrete far from bearing plate hole is low. When PBL connector is in compression zone, it will make full use of its shear capacity to a greater degree.

\section{Introduction}

There is full size or scale model test method and numerical analysis method to analyze the shear performance of connector in non-steel-cell joint section of hybrid girder bridge. And now, there is no uniform analysis method to simulate the characteristics of concrete materials and the boundary conditions of the joints and concrete among numerical analysis method. Considering the nonlinear properties of structural materials and nonlinear relationship between the contact hole and concrete tenon [1,2], this paper taken non-steel-cell joint section of Zhejiang hybrid girder bridge as the research object and the finite element model of joints was established to analyze the shear performances.

\section{Modeling}

Referring to the PBL connector and corresponding boundary conditions of Changqiao Bridge, the paper chose the PBL connector, filled concrete and steel structure of the joint in the centerline of the bridge as the research objects. Based on the nonlinear characteristics of materials, the local model of PBL connector was established to analyze the shear behavior in the combination section [3]. The location of PBL connector of Changqiao Bridge is shown in Fig.1. 


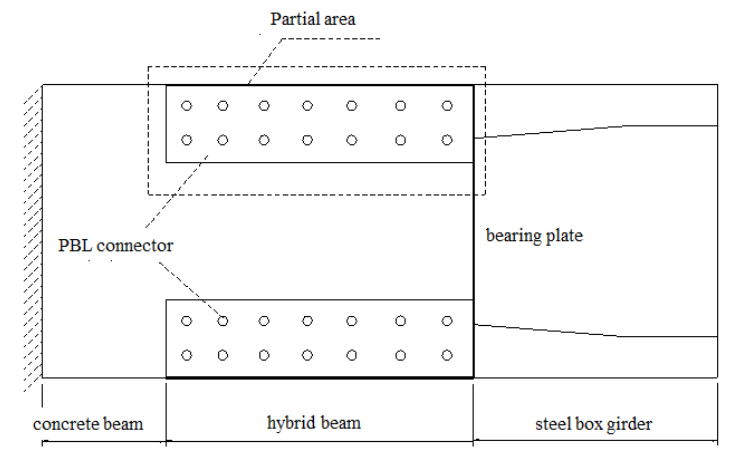

Fig.1 The location of PBL connector of Changqiao Bridge

The finite element analysis models including PBL connector, steel roof, rear bearing plate, part of the concrete beams, concrete tenon and steel rebar were established. Q345C and C50 were selected as the analysis material in the model. The thickness of the PBL connector is $20 \mathrm{~mm}$, the radius of the hole is $30 \mathrm{~mm}$ and the diameter of the steel rebar is $20 \mathrm{~mm}$. Size of connector and finite element simulation model are shown in Fig.2 to Fig.6.

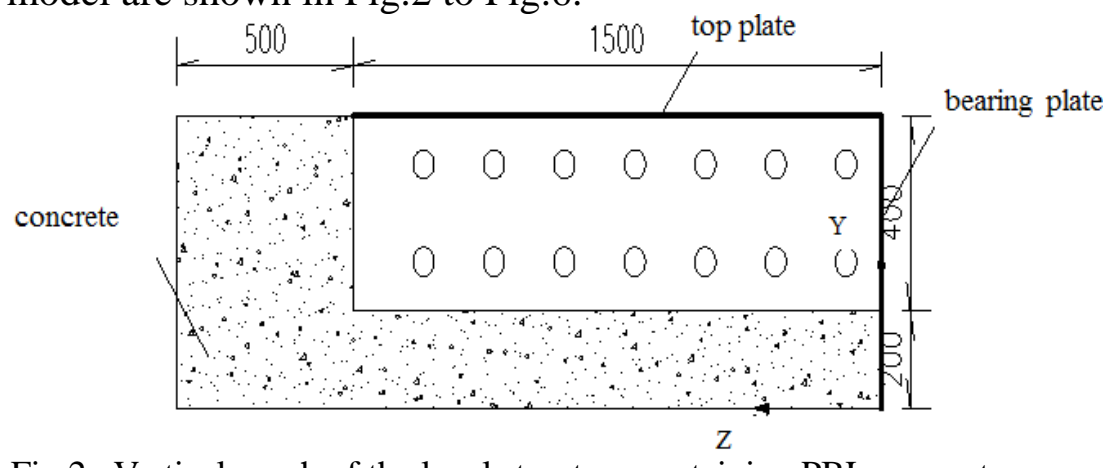

Fig.2 Vertical graph of the local structure containing PBL connector

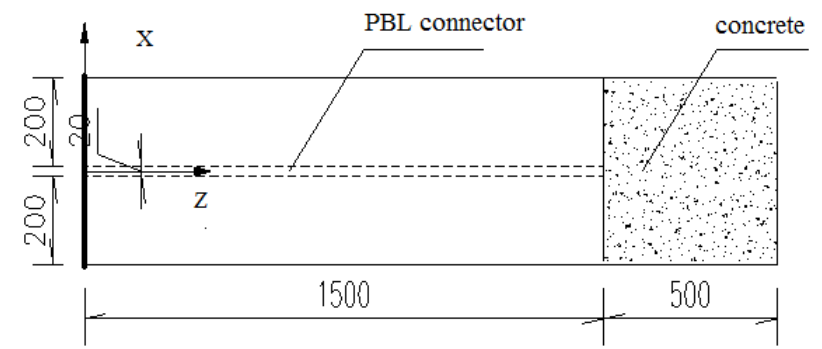

Fig.3 Plane graph of the local structure containing PBL connector

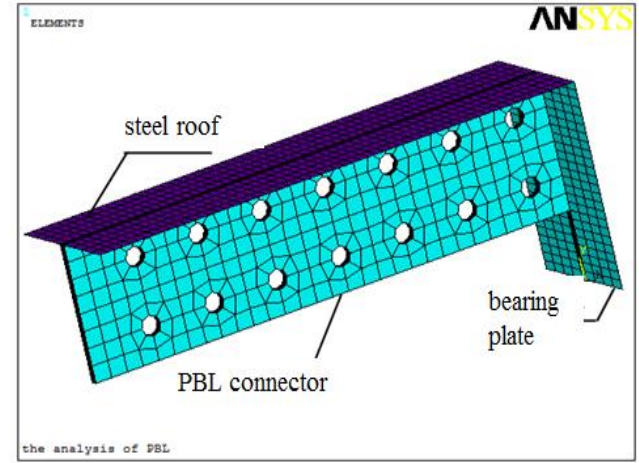

Fig.4 Steel structure in model

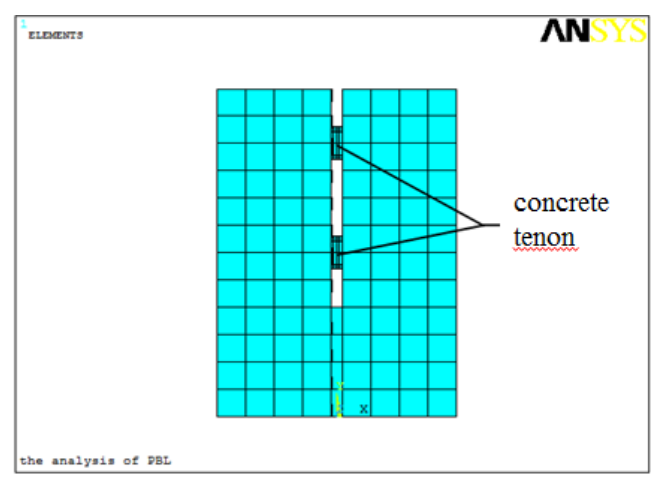

Fig.5 Concrete structure in model 


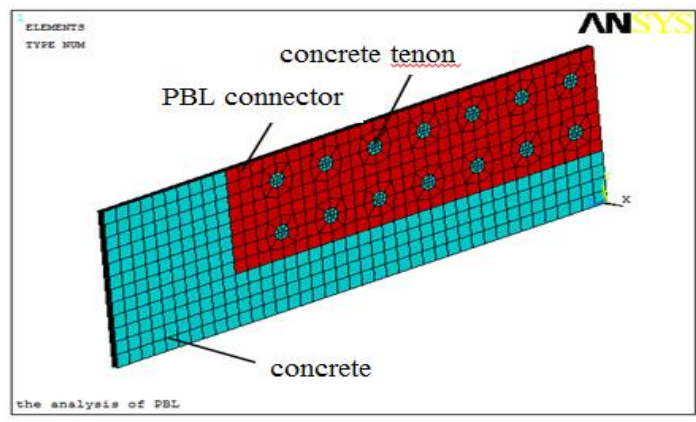

Fig.6 PBL connector and concrete in model

In the model, the solid45 element is adopted in the connector; the beam188 is used in the steel rebar; the shell63 is used in the bearing plate and top and bottom slab of the mixed section steel beam; the solid65 is used in the concrete which is in contact with PBL connector, where nonlinear characteristics of materials is considered.

Research focuses on the shear bearing capacity and stress state of the PBL connector. The concrete surface at $\mathrm{Z}=2.0 \mathrm{~m}$ is consolidated in the model. Top of the PBL connector and the roof girder, root and bearing plate are coupled on displacement coordinates to simulate welding. Taking into account the connection between the concrete and the two sides of connector, normal line displacement of both sides are restricted to prevent deformation out of surface during loading. And side plane direction of the connector is free. Because the chemical bonding effect and friction between the connection and concrete are ignored, so the calculation of ultimate bearing capacity of PBL connector is conservatively. Assuming the bearing plate is in close contact with concrete filled in the mixed section, both nodes on surface are coupled to form complete shear connection [4].

Suppose that hybrid beam combination section meets the plane assumption, so there is a linear relationship between the normal stress and the centroid axis distance when the section is under bending load. When hybrid beam is under bending load, its top plane is in compression or tension. $\mathrm{q}\left(\right.$ unites: $\mathrm{kN} / \mathrm{m}^{2}$ ) here is used to represent load intensity in top plane, which is shown in Fig.7 to Fig.8.

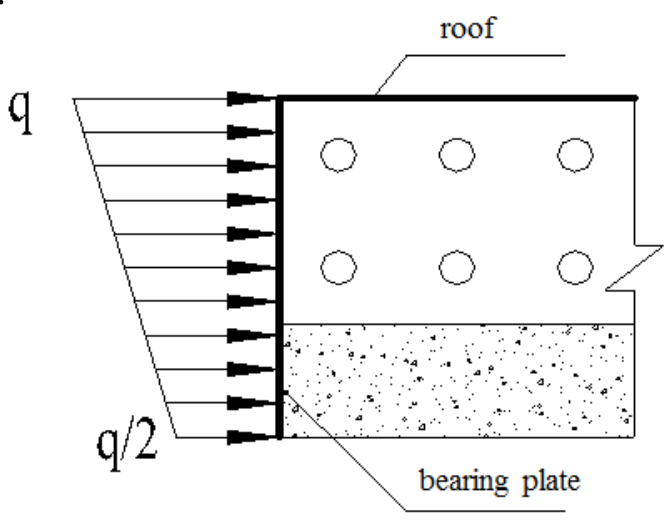

Fig.7 PBL connector in compression

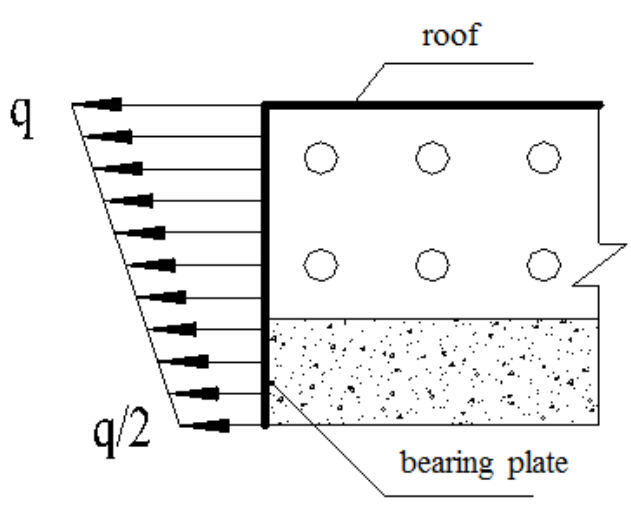

Fig.8 PBL connector in tension

\section{Mechanical behaviors of PBL connector}

PBL connector in tension. When the PBL connectors is in tension, the distal end of the connector would separate from the concrete, if we ignore the bonding property between the distal end $(\mathrm{Z}=1.5 \mathrm{~m})$ and the bottom $(\mathrm{Y}=-0.6 \mathrm{~m})$ of the connector and the concrete. The negative $\mathrm{Z}$ direction gradient surface load is applied on the surface of the bearing plate. When the load is small, concrete and steel are in the elastic state, and the bearing plate transmits the force to connector from the beginning to the end, so that the deformation of steel connector occurs along the negative $\mathrm{Z}$ axis. Concrete tenons limit deformation of connector and the stress is transferred to the concrete 
tenon by the wall of connector holes, then the stress is transmitted to concrete by the compressive tenon, which forms an effective shear mechanism [5].

With the increase of load, the cracks appear in the concrete which is near the bearing plate. The shear cracks at the first and second column tenons begin to appear, and gradually transmit to the third and other column tenons.

When the load intensity is $-4000 \mathrm{kN} / \mathrm{m}^{2}$, the sides of the first column concrete tenon are full of cracks, and the tenons are in shear fracture state; when the load intensity is up to $-6500 \mathrm{kN} / \mathrm{m}^{2}$, the first and second column tenons are completely broken, and the principal compressive stress of the first column tenon is higher than the design value of concrete compressive strength. However, the degree of cracks on the third to fifth column tenon are lower, and stress of other column concrete tenon is still not high.

Higher the load intensity, severer the cracks, higher the Von Mises of steel. The Von Mises on connector in the distal is lower than near-end. Fig.9 shows Von Mises stress of connector on different height when the load intensity is $-6500 \mathrm{kN} / \mathrm{m}^{2}$. PBL connector's Von Mises stress is shown in Fig.10. Concrete's Von Mises stress is shown in Fig.11.

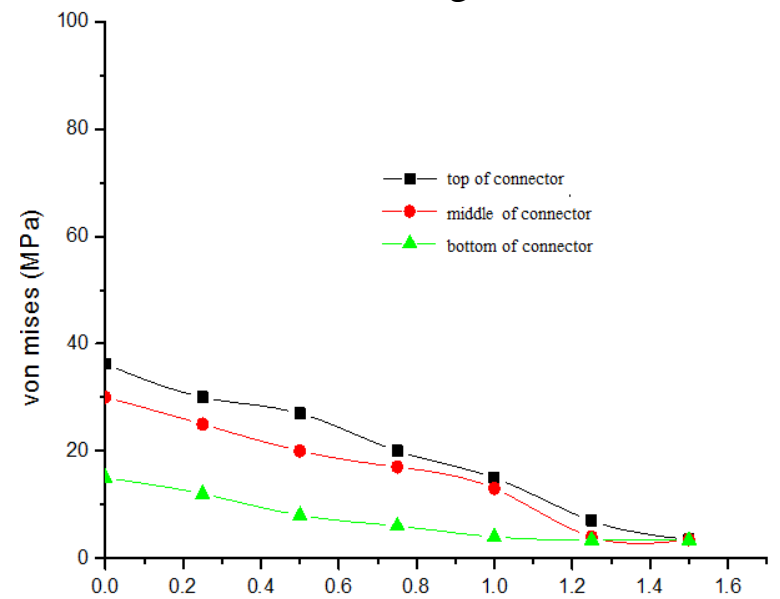

Fig.9 Von Mises stress of connector on different height under ultimate load

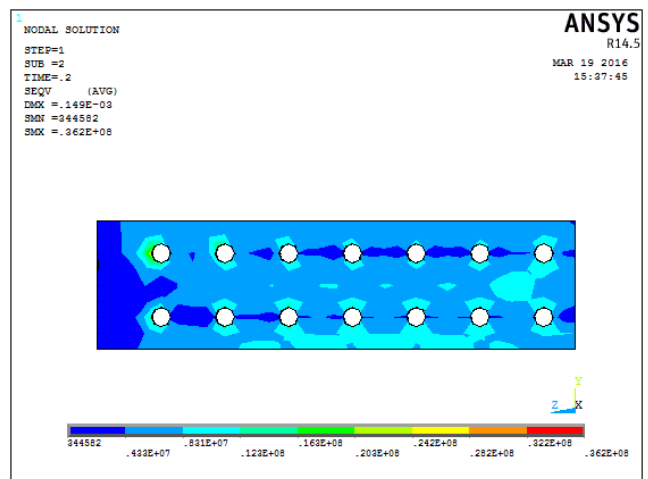

Fig.10 Von Mises stress nephogram of connector in tension under ultimate load

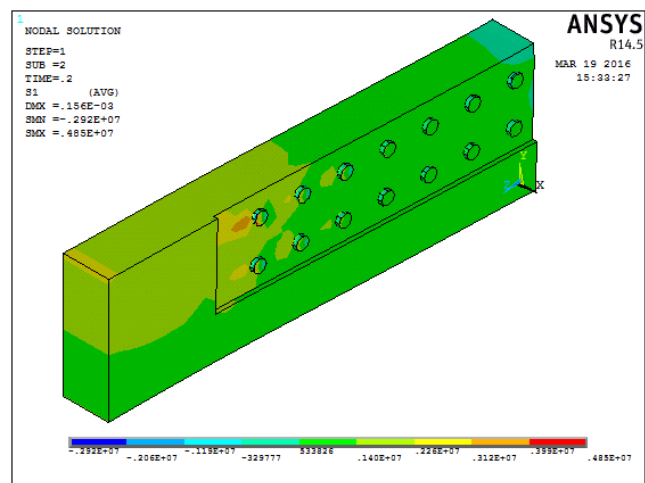

Fig.11 Principal tensile stress of concrete in tension under ultimate load

The calculation results of the PBL connector in tension show that when the load intensity reaches $-6500 \mathrm{kN} / \mathrm{m}^{2}$, partial destructions of the first and second column concrete tenon will affect the continuous shear resistance of the model.

PBL connector in compression. When PBL connectors is in compression, there is a transfer of compressive stress between the distal end of connector and concrete. And this model is different from that in tension, so contact element $(\mathrm{Z}=1.5 \mathrm{~m})$ was set to transfer compressive stress from the distal end of connector to concrete [6,7]. When the flange is in tension, connector holes and concrete tenon which are near to the bearing plate are first to participate into work until the 
concrete tenon are broken , and then the connector holes and concrete tenon at distal ends will be in a low stress state. When PBL connectors is in compression, the deformation of PBL connector will be limited by concrete, so concrete tenon and PBL connector at distal ends will participate into work earlier.

The ANSYS model is loaded step by step in slope load. The stress and deformation in each load step are viewed through the software. When the load is small, concrete and steel are in elastic state, the deformation of concrete near bearing plate in $\mathrm{Z}$ direction is larger than that of the connector. Due to the connection holes, the deformation of steel is same as the concrete with the pressure transmitted to the central connector.

When the load intensity is $5500 \mathrm{kN} / \mathrm{m}^{2}$, the tiny cracks in tensile side begin to appear due to the shear stress of concrete tenon. When the load intensity is up to $7000 \mathrm{kN} / \mathrm{m}^{2}$, the cracks expand in small scale. However, the compressive stress of the concrete tenon does not exceed the axial compressive strength of concrete material, so there is no crack in compression side. When the load intensity is increased to $11000 \mathrm{kN} / \mathrm{m}^{2}$, three columns of middle concrete tenon are broken from compression, and there are a large number of cracks on both sides of concrete. Fig.12 shows the peak principal compressive stress when the load intensity is $11000 \mathrm{kN} / \mathrm{m}^{2}$, and Fig.13 to Fig.14 show the stress nephogram of concrete and connector in compression.

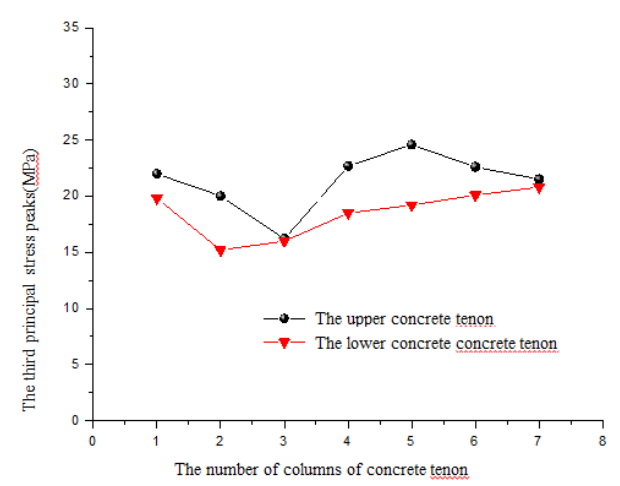

Fig.12 Stress distribution of concrete under compression limit load

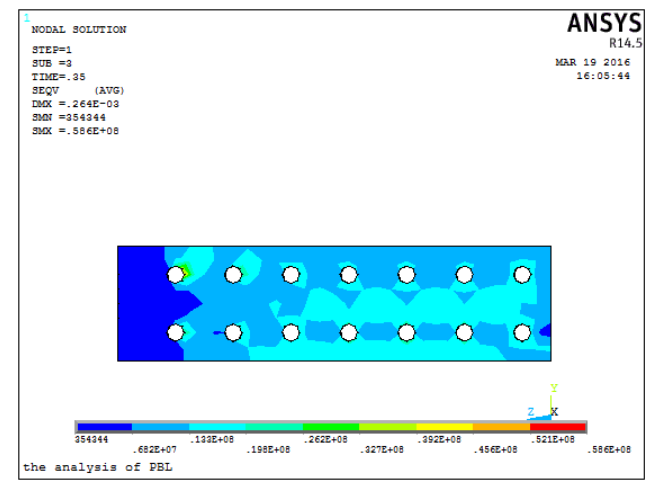

Fig.14 Equivalent stress of connector when the load intensity is $11000 \mathrm{kN} / \mathrm{m}^{2}$

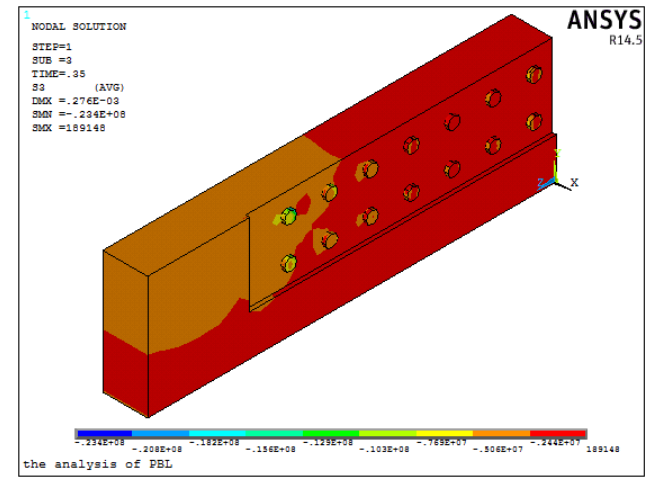

Fig.13 Main compressive stress of concrete when the load intensity is $11000 \mathrm{kN} / \mathrm{m}^{2}$

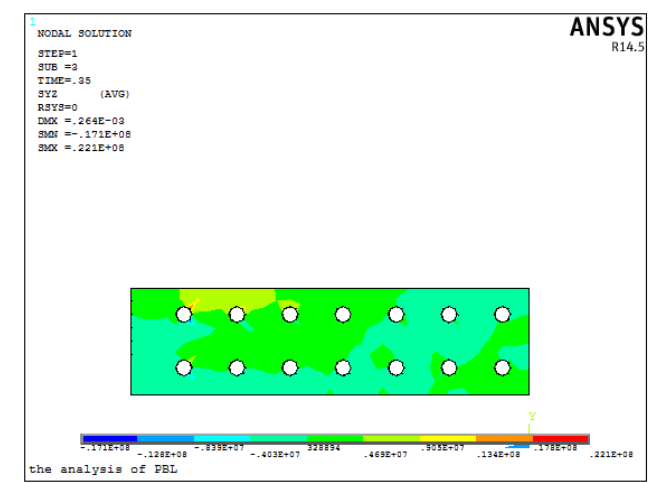

Fig.15 Shear stress of connector when the load intensity is $11000 \mathrm{kN} / \mathrm{m}^{2}$

As can be seen from Fig.1 to Fig.15, the stress distribution of PBL connector in compression is more uniform than that in the tension when the concrete tenon is in work. The principal compressive stress of the fourth and fifth column concrete tenon is slightly larger, and the ultimate compressive surface load is larger than the tensile one. In same dimensions and material properties, PBL connector provides greater shear bearing capacity under the compression condition . 


\section{Conclusions}

Steel-concrete composite beam segment is a key structure of the hybrid beam, which is related to the safety, reliability and durability. The nonlinear properties of the material and the contact relationship which just transfer the pressure between the PBL connector and concrete were considered, and the results from the shear behavior analysis of the PBL connectors are as follows:

When the PBL connector is located in the tension zone and the load intensity is up to $-6500 \mathrm{kN} / \mathrm{m}^{2}$, the first and second column concrete tenon are seriously damaged and the continuous shear resistance is affected. However, the Von Mises stress of the sixth and seventh concrete tenon is small, and there is still a large surplus in the shear resistance of the shear key.

When the PBL connector is located in the compression zone and the load intensity is close to $11000 \mathrm{kN} / \mathrm{m}^{2}$, three columns of middle concrete tenon are broken from compression, and there are a large number of cracks on both sides of concrete. The peak compressive stress of each column concrete is reaching the principal compressive stress, so the concrete tenons are fully utilized.

\section{References}

[1] JIANG Yu-song, SU Chao. Numerical Analysis Methods for Solving Contact Problems in Engineering [J]. Water Power, 2010, 04:75-78. (In Chinese)

[2] HAN Qing, ZHANG Yi-gang, ZHAO Kai-hong. A Numerical Method of the Contact Problems in Structural Engineering [J]. Journal of Beijing University of Technology, 2006, 04:321-326. (In Chinese)

[3] The People's Republic of China Ministry of Housing and Urban-rural Development, GB50010-2010, Code For Design of Concrete Structures [S].Beijing: China Building Industry Press, 2011-07-01. (In Chinese)

[4] YAO Guo-wen, ZHOU Duan-ming, ZHOU Zhi-xiang. Study on Stress Transfer in Steel-Concrete Joint of Hybrid Arch Bridge [J]. Journal of Chongqing Jiao Tong University Natural Sciences, 2008, 02:177-180. (In Chinese)

[5] XU Yan, WANG Yi, LIU Ping. Study on Calculation Formula of Bearing Capacity of Opening Steel Plate Joint [J]. Building Science Research of Sichuan, 2010, 05:30-33. (In Chinese)

[6] Vianna J, Andrade S, Vellasco P, et al. Experimental Study of Perfo-Bond Shear Connectors In Composite Construction [J]. Journal of Constructional Steel Research, 2013, 81: 62-75.

[7] CHEN Ke, Song Jia-yong, Etc. Simulation and Analysis for Externally Prestressed Concrete Bridge Based on ANSYS [A].1st International Conference on Civil Engineering, 2011. 